\title{
Development in fission track-thermal ionization mass spectrometry for particle analysis of safeguards environmental samples
}

\author{
C. G. Lee, ${ }^{1 *}$ K. Iguchi, ${ }^{1}$ J. Inagawa, ${ }^{1}$ D. Suzuki, ${ }^{1}$ F. Esaka, ${ }^{1}$ M. Magara, ${ }^{1}$ S. Sakurai, ${ }^{1}$ \\ K. Watanabe, ${ }^{2}$ S. Usuda ${ }^{1}$
}

${ }^{1}$ Nuclear Science and Engineering Directorate, Japan Atomic Energy Agency (JAEA), Tokai-mura, Naka-gun, Ibaraki 319-1195, Japan

${ }^{2}$ Planning and Coordination Office, Japan Atomic Energy Agency (JAEA), Tokai-mura, Naka-gun, Ibaraki 319-1195, Japan

appeared in the Journal of Radioanalytical and Nuclear Chemistry, Vol. 272, No. 2 (2005) 299-302.

On page 300, Fig. 1 should read:

a)

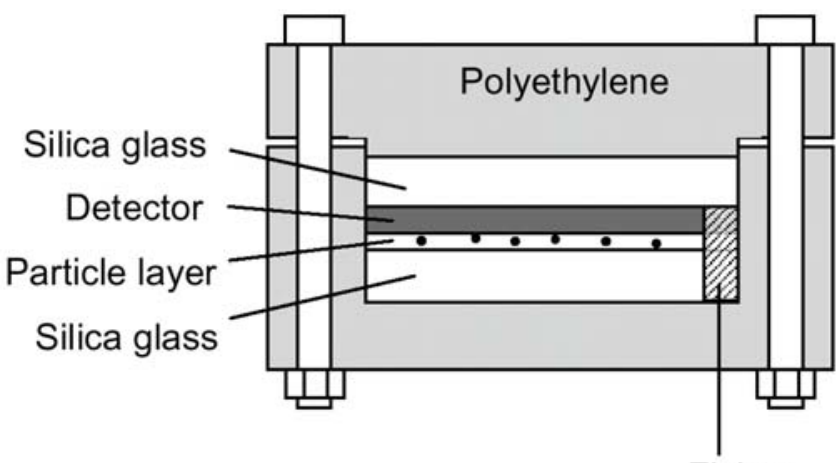

Fixing

b)

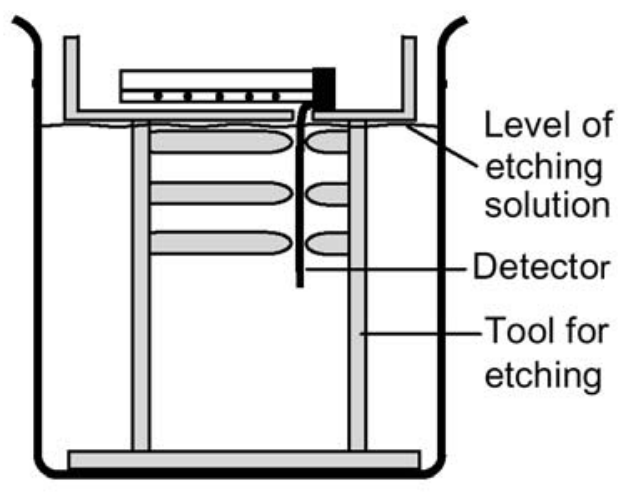

$20 \mathrm{~mm}$

c)

Observation with

digital microscope

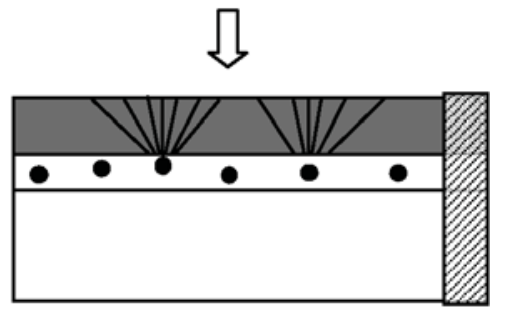

Fig. 1. Schematic diagram of the particle layer, detector, and silica glasses inside an irradiation capsule (a), schematic side view of the tool made of silica glass for exclusively etching a detector (b), and the method of identification of the particle containing fissile materials from fission tracks (c) 